while the patient attempted left lateral gaze. Vertical eye movements were normal, and no ocular bobbing was observed. There was no long tract sign, and the cerebellar system was normal. The remainder of the examination was unremarkable.

ISOLATED ONE AND A HALF SYNDROME: AN

\section{ATYPICAL PRESENTATION OF NEUROCYSTICERCOSIS}

Sir,

There are many reports on ocular motility disturbances-caused by neurocysticercosis of brainstem ${ }^{[1-3]}$ and fourth ventricular neurocysticercosis cyst $^{[4]}$ in the available literature, but isolated one and a half syndrome due to neurocysticercosis has not been reported. We describe a patient with one and a half syndrome without any other brainstem signs, caused by neurocysticercosis involving the pons.

An 18-year-old male presented with a history of sudden-onset diplopia of 1-week duration and blurring of vision. He had no history of headache, fever, seizures, walking difficulty, previous head injury, diabetes or hypertension. There was no history of a similar episode in the past. Diplopia was found increased while looking to the left. Findings from general examination were normal. The left eye was deviated outward, and the right eye was central in primary gaze. There was weakness of adduction in the left eye and paralysis of all conjugate horizontal eye movements in the right eye. The left eye also showed nystagmus
Findings from routine blood examination, including blood biochemistry and chest skiagram, were normal. Mantoux test was negative. Cerebrospinal fluid (CSF) analysis revealed a clear fluid with leukocyte count of 2/mm3; protein, $25 \mathrm{mg} / \mathrm{dL}$; and CSF glucose, more than $50 \%$ that of serum. Findings from direct examination and culture of CSF were negative for acid-fast bacilli fungal and bacterial pathogens. Computed tomography (CT) and magnetic resonance imaging (MRI) sections showed a small ring-enhancing lesion in the dorsomedial pons [Figures 1 and 2]. CSF for adenosine deaminase assay was negative, and enzyme-linked immunoabsorbent assay for anticysticercal antibodies (IgM and $\lg$ ) in cerebrospinal fluid was positive. A diagnosis of neurocysticercosis was made, and the patient was treated with albendazole and dexamethasone. The patient showed

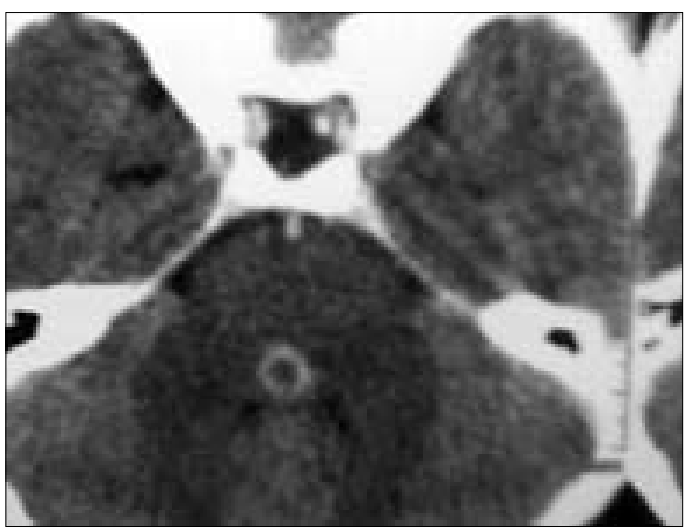

Figure 1: CT scan of the brainstem showing a granuloma in the dorsomedial pons 


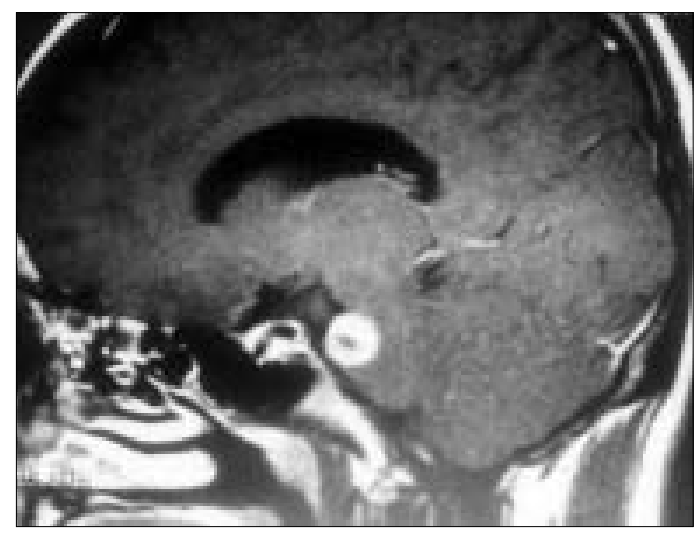

Figure 2: T1 weighted MRI image showing pontine granuloma with eccentric nidus

improvement in symptoms within 2 weeks. Follow-up after 3 months showed he was symptom-free with no neurological deficit; repeat $\mathrm{MRI}$ brain was advised but the patient refused.

The present case describes a patient with one and a half syndrome due to pontine granuloma (neurocysticercosis). In one and a half syndrome, the lesion affects the paramedian pontine reticular formation (PPRF) (or the abducens nucleus) and the medial longitudinal fasciculus (MLF) on the same side (the MLF having crossed from the opposite side). It is most often caused by multiple sclerosis, brain stem stroke, brain stem tumors, arteriovenous malformations and infective mass lesions such as tuberculomas. ${ }^{[5]}$ The present case is very unusual because one and a half syndrome was the only abnormality in this patient with pontine neurocysticercosis, because of the unique position of the MLF and PPRF within the brain stem. So, in a highly endemic area like India, neurocysticercosis should be considered as a differential diagnosis of one and a half syndrome.
M. P. RANJITH, R. DIVYA, ANUPAM SAHNI Department of Medicine, Netaji Subhash Chandra Bose Medical College, Jabalpur, India

Correspondence: Dr. Ranjith M. P. Bhavatharini, P.O. Pantheerankave, Kozhikode, Kerala - 673 019, India. E-mail: drranjithmp@gmail.com

\section{REFERENCES}

1. Ranjith MP, Divya R, Sahni A. Isolated third cranial nerve palsy: A rare presentation of neurocysticercosis. Ir J Med Sci 2009 In press.

2. Singh NN, Verma R, Pankaj BK, Misra S. Neurocysticercosis presenting as Weber's syndrome: As short report. Neurol India 2003;51:551-2.

3. Garg RK, Karak B. Neurocysticercosis presenting as midbrain syndrome. J Assoc Physicians India 2000;48:533-5.

4. Keane JR. Internuclear ophthalmoplegia: Unusual causes in 114 of 410 patients. Arch Neurol 2005;62:714 -7.

5. Vimla M, Madhurjya G, Rohit S, Sumit S, Arvind $\mathrm{K}$. Isolated "one and a half syndrome" with brain stem tuberculoma. Indian J Pediatr 2004;71: 469-71.

DOI: 10.4103/0019-5359.49291 